\title{
Systematic Delineation of Media Polarity on COVID-19 Vaccines in Africa: Computational Linguistic Modeling Study
}

Sefater Gbashi ${ }^{1 *}, \mathrm{PhD}$; Oluwafemi Ayodeji Adebo ${ }^{1 *}, \mathrm{PhD}$; Wesley Doorsamy ${ }^{2 *}, \mathrm{PhD}$; Patrick Berka Njobeh ${ }^{1 *}, \mathrm{PhD}$

${ }^{1}$ Faculty of Science, University of Johannesburg, Johannesburg, South Africa

${ }^{2}$ Institute for Intelligent Systems, University of Johannesburg, Johannesburg, South Africa

*all authors contributed equally

Corresponding Author:

Sefater Gbashi, PhD

Faculty of Science

University of Johannesburg

55 Beit Street, Doornfontein

Johannesburg, 2028

South Africa

Phone: 27620402580

Email: sefatergbashi@gmail.com

\section{Abstract}

Background: The global onset of COVID-19 has resulted in substantial public health and socioeconomic impacts. An immediate medical breakthrough is needed. However, parallel to the emergence of the COVID-19 pandemic is the proliferation of information regarding the pandemic, which, if uncontrolled, cannot only mislead the public but also hinder the concerted efforts of relevant stakeholders in mitigating the effect of this pandemic. It is known that media communications can affect public perception and attitude toward medical treatment, vaccination, or subject matter, particularly when the population has limited knowledge on the subject.

Objective: This study attempts to systematically scrutinize media communications (Google News headlines or snippets and Twitter posts) to understand the prevailing sentiments regarding COVID-19 vaccines in Africa.

Methods: A total of 637 Twitter posts and 569 Google News headlines or descriptions, retrieved between February 2 and May 5, 2020, were analyzed using three standard computational linguistics models (ie, TextBlob, Valence Aware Dictionary and Sentiment Reasoner, and Word2Vec combined with a bidirectional long short-term memory neural network).

Results: Our findings revealed that, contrary to general perceptions, Google News headlines or snippets and Twitter posts within the stated period were generally passive or positive toward COVID-19 vaccines in Africa. It was possible to understand these patterns in light of increasingly sustained efforts by various media and health actors in ensuring the availability of factual information about the pandemic.

Conclusions: This type of analysis could contribute to understanding predominant polarities and associated potential attitudinal inclinations. Such knowledge could be critical in informing relevant public health and media engagement policies.

(JMIR Med Inform 2021;9(3):e22916) doi: 10.2196/22916

\section{KEYWORDS}

COVID-19; coronavirus; vaccine; infodemiology; infoveillance; infodemic; sentiment analysis; natural language processing; media; computation; linguistic; model; communication

\section{Introduction}

\section{COVID-19}

COVID-19, a communicable disease caused by SARS-CoV-2, has resulted in the ongoing COVID-19 pandemic [1], with significant health and socioeconomic effects in almost all countries of the world, mainly due to the shutdowns, social distancing, and the resultant business interruptions [2-5]. On March 11, 2020, the World Health Organization (WHO) declared a COVID-19 outbreak due to its global adverse (health and economic) impact [6,7], which is still unfortunately escalating as the world eagerly awaits a medical solution. According to projections by the International Monetary Fund, 
global economic growth could fall by $0.5 \%$ for the year 2020 due to the impact of COVID-19 [7]. A United Nations study revealed that, for the first time since 1900, global poverty could increase [8]. The world unemployment rate has been estimated to hit a mark of $9.4 \%$ by the end of 2020 , in contrast to the $5.3 \%$ seen in 2019 [9]. A forecast by the World Bank projects that Africa is heading toward its first recession in the last 25 years, being driven by the impact of COVID-19 [2]. There is a compelling necessity to achieve an immediate medical breakthrough to curtail the pandemic. Although currently there is no known cure or vaccine for COVID-19, various agencies and governments are working round the clock to proffer much needed solutions. There are indications that some of the vaccines could be ready for clinical trials soon, while others are already undergoing trials.

\section{Infodemic and COVID-19}

Amid the spread of the COVID-19 pandemic and the efforts to provide a solution, there has equally been substantial media interest and widespread misinformation on the web about the scale, origin, diagnosis, prevention, and treatment of the disease [10]. The WHO has expressed particular concern about this infodemic and its impact on the fight against the COVID-19 pandemic [11-13]. This "infodemic" may have been facilitated by the global lockdown, isolation, and social distancing situation worldwide, which has led to increased internet and social media use [14,15]. For example, as of May 2020, global internet use was already up by $7 \%$ (since the same time in 2019), while the number of social media users increased by $8 \%$ (reaching 3.81 billion) since April 2019 [14]. Rozenblum and Bates [16] described the internet and social media as the "perfect storm" with regard to patient-oriented health. Singh [17] called the COVID-19 pandemic-induced media use a "compulsive social media use" and wonders whether it is an addictive behavior. Information diffusion and media coverage can have a positive effect on an epidemic by shortening the duration of the outbreak and reducing its burden [18]; however, if media communication is not properly managed, this can have a negative effect $[19,20]$. In a study by Brainard and Hunter [21], the authors noted that making $20 \%$ of a population unable to share fake advice or reducing the amount of damaging advice spreading online by just $10 \%$ can reduce the severity of a disease outbreak.

It is known that medical interventions involving infectious diseases and vaccines in particular are usually predisposed to infodemics [22-24]. Indeed, disease proliferation and associated potential effects take place in a dynamic social space, wherein public knowledge, sentiments, and individual health decisions are influenced by the media and cultural norms [19,25,26]. Studies have shown that an important factor in determining the success of a medical intervention, particularly a vaccination program, is the degree of public acceptance of the proposed intervention [27-29]. Lewandowsky et al [30] observed that the spread of myths regarding vaccinations has led to more parents being reluctant to adopt such measures. The withdrawal of the first US Food and Drug Administration licensed vaccine against the rotavirus in 1999 has been strongly correlated with negative media coverage [31]. Infodemics are known to foster the denial of scientific evidence [32]; incite apathy, cynicism, and extremism [33]; and cause people and institutions to take actions that may not necessarily be in their best interests or for the good of society in general $[19,34]$. Yu et al [19] observed that, even though medical interventions were able to reduce hepatitis B virus infection by up to $90 \%$ among children younger than 5 years using effective and safe hepatitis B (HepB) vaccines, negative media reports about infant death after HepB vaccination resulted in a loss of confidence in vaccines and an approximately $19 \%$ decrease in the use of vaccines within the monitored provinces in China. This further emphasizes the importance of individuals' actions in controlling the spread of the disease and the role of the media and psychosocial effects in this regard, as also observed in the literature $[12,13,25,35]$.

In recognizing the importance of information and corresponding human actions in the management of diseases and other medical emergencies or outbreaks, medical researchers are increasingly exploring the application of computer and mathematical models to analyze communications relating to health problems and to derive value from such resources [18,25,35-37]. One such application is sentiment analysis [38,39]. It is important to understand the prevailing emotions in public media communications, as sentiments could provide a richer set of information about people's choices and reactions, and in many cases, even determine their decisions [40-42].

\section{Sentiment Analysis}

Sentiment analysis is a multidisciplinary field involved with machine learning (ML) and artificial intelligence (AI), and a subset of natural language processing (NLP) concerned with the systematic extraction, analysis, classification, quantification, and interpretation of affective tonality, opinions, and subjective information in human communications (written or spoken) using computational linguistic methods to derive value of the opinions people express. Sentiment analysis is important because opinions and emotions constitute a critical aspect of humans and play a key role in perceptions of reality, choices, actions, and behaviors [37]. Basic tasks in sentiment analysis include classifying emotional polarity in a communication as positive, negative, or neutral and often scaling such polarities to reflect the depth of the expressed emotions. By understanding prevailing sentiments in communications, health actors can then make more relevant and informed decisions of public relevance and act accordingly to improve the medical experience.

In performing sentiment analysis, language limitations remain a major challenge, as most of the sentiment analysis models and libraries are built in English, which limits their applicability for texts written in other languages. Another problem with traditional sentiment analysis models is the lack of context in labeling lexical items and extracting features, which results in ambiguity in polarity representations. A word or group of words can have different meanings and polarities in different contexts; hence, the global representation of words and sentences could influence the semantics of the words [43]. More contemporary developments in sentiment analysis are shifting toward increasingly context-based sentiment analysis and applicability in other languages [43-45]. Context-aware (ie, context-based) sentiment analysis attempts to tackle the challenge of ambiguity by taking into account all of the text around any given word or words, then processing the logical structure of the sentences, 
establishing the relations between semantic concepts and assigning logical grammatical roles to the lexical elements to decipher the most relevant meaning of a word or group of words that have more than one definition.

Generally, sentiment analysis models can be largely grouped into two main categories: lexical rule-based techniques and learning-based approaches [43]. The former (ie, lexical rule-based methods) involve the use of predefined lexicons annotated with sentiment polarities, "positive," "negative," or "neutral," which are then used to determine the sentiment of the analyzed text [46]. Lexical rule-based sentiment analyzers such as TextBlob and Valence Aware Dictionary and Sentiment Reasoner (VADER) [47,48], although they use predefined dictionaries, take in to account punctuation such as exclamation marks, emoticons, capitalizations (which gives added intensities to assigned polarities), and negation words (which reverse the polarities). Lexical rule-based sentiment methods have the advantage of being easy to implement because there is no need for the exhausting and arduous task of tagging texts for training. In addition, the approach prevents overfitting because the lexicons are predefined independently of the data being analyzed, a feature that also enables it (lexical rule-based sentiment models) to be used on multiple data sets [46]. On the other hand, the main advantage of (machine) learning-based methods is that they enable the use of domain-specific data sets to train the models, which are then used to analyze a given text. Given relevant and adequate training data sets, this (domain-oriented training) substantially increases the accuracy and level of confidence in text classification and sentiment analysis using learning-based methods. Examples of successful implementation of ML-based sentiment models can be found in the literature [49].

\section{Applications of Sentiment Analysis}

Sentiment analysis has been applied in the fields of medical research, political science, business, mass communication, and education. Specific applications include brand monitoring and reputation management, customer feedback and support, product analysis, market research and competitive research, competitor analysis [50], attitudinal analysis [51], medical records analysis, patient experience analysis, and infodemiology [52-54]. More than ever before, the application of sentiment analysis in the medical field has seen a surge, as both patients and medical practitioners increasingly use web-based platforms such as websites, social media, and blogs to search for treatment-related information and to convey opinions on health matters $[55,56]$. Melzi et al [57] implemented a supervised learning model to extract sentiments from forums of Spine Health to retrieve patient knowledge. Yang et al [58] demonstrated a sentiment analysis-based framework to derive insights from user-generated content from health social media.

In the context of the ongoing COVID-19 pandemic, Barkur and Vibha [59] performed a sentiment analysis of Indians' Twitter communications about the nationwide lockdown due to the COVID-19 outbreak. An investigation of the sentiment and public discourse during the pandemic was performed using latent Dirichlet allocation for topic modeling on 1.9 million Tweets written in the English language related to COVID-19 collected from January 23 to March 7, 2020 [60]. Deep long short-term memory models were used to scrutinize the reaction of citizens, public sentiment, and emotions from different cultures about COVID-19 and the subsequent actions taken by different countries [61]. These studies reiterate the usefulness of sentiment analysis in the health domain. Nonetheless, there is no study on the sentiment analysis of media communications regarding COVID-19 vaccines in Africa that can guide health actors to understand the prevailing polarities and to make relevant polices, as the continent awaits the availability of viable vaccines to combat the pandemic.

\section{Research Justification, Goal, and Questions}

Indeed, opinion mining and sentiment analysis in public media is a daunting task because of the large amount of information shared on the internet and social media, particularly in recent times. To give some perspective, Google handles approximately 4.5 million queries every 60 seconds, and approximately 500 million tweets are made every 24 hours [62,63]. In the month of March 2020 alone, about 550 million tweets were made, which included the terms COVID-19, COVID19, COVID_19, coronavirus, corona virus, or pandemic, according to the Pan American Health Organization [64]. Considering the enormity of internet information, researchers are increasingly adopting computer-based algorithms to process, analyze, and interpret such data. This does not only substantially limit the need for human power but also eliminates the associated human biases and inefficiencies in many ways $[65,66]$.

The goal of this paper is to retrieve timely and relevant information that is publicly available regarding COVID-19 vaccines in Africa and interrogate such resources in an attempt to extract the sentiment polarities using computational linguistics models. In this regard, we outlined the following research questions, which constitute the main contributions of this paper:

- What was the media activity patterns regarding COVID-19 vaccines in Africa within the study period of February 2 to May 5, 2020?

- What are the prevailing sentiments (ie, positive, negative, or neutral) in the communications?

- How does the sentiment polarities in Twitter posts compare to those in Google News communications?

- Using three different sentiment analysis approaches, how does the sentiment results from these models compare with each other?

- What were the specific activities or events that might have triggered the prevailing emotions in the communications?

\section{Methods}

This study adopts computational linguistic models (TextBlob, VADER, and Word2Vec-bidirectional long short-term memory [BiLSTM]) to scrutinize communications from popular web-based media sources regarding COVID-19 vaccines in Africa.

\section{Data Collection and Cleaning}

In the collection of data, the following criteria and procedure were used [67]. 


\section{Data Source}

Twitter and Google News were selected as the data sources. This is because both media outlets are important web-based information sources for many people. Von Nordheim et al [68] examined the use of different social media platforms (ie, Twitter and Facebook) as journalistic sources in newspapers of three different countries; the authors observed that Twitter is more commonly used as a news source than Facebook, and in comparison to Facebook, Twitter was primarily used as an elite channel [68]. In addition, previous studies have shown that most of the communications on the Twitter platform are truthful, although sometimes it may also be used to propagate false information and rumors $[67,69,70]$. Moreover, the microblog (ie, Twitter) facilitates the propagation of real-time information to a large group of people, making it an ideal tool for the dissemination of breaking news [69]. Twitter has become a favored communication platform for various social protest movements [71]. On the other hand, Google News is an online media service that aggregates and presents a continuous flow of news content from more than 20,000 publishers worldwide and is available on the web, iOS, and Android. For news in the English language, it covers about 4500 sources. This creates a unique information space, providing a major gateway for consumers to access news, reducing the time and effort needed to regularly check different media sources for updates, and increasing overall user engagement.

\section{Query Terms}

English language keywords were used to query the data sources instead of hashtags. Specific search terms used were "COVID-19 vaccine Africa," "COVID19 vaccine Africa," and "Coronavirus vaccine Africa." This approach is more inclusive, as it includes communications that have hashtags of the keywords [67]. Moreover, hashtags are often short-lived on the web.

\section{Query Period}

The sources were queried within the period of February 2 to May 5, 2020, using the search terms. The study period was chosen on the basis of timelines, relevance, and particularly because this was the time frame in which opinions, perspectives, and narratives about the search terms were emerging and could set the tone for subsequent communications as well as make enduring impressions on people's dispositions.

\section{Search Tools}

Google News data was retrieved using the Google News application processing interface (API) for Python by $\mathrm{Hu}$ [72]. Twitter data were obtained by manually scraping the Twitter web application for publicly available English language posts containing the query terms. This was essentially due to limited resources to subscribe for the Twitter API, which is a paid service. To eliminate any biases, a new Twitter account was used with no previous search history, liked pages, or topics or interests associated with the account.

A total of 569 news headlines and snippets published in English were obtained from Google News and saved as a comma-separated values (CSV) file (Multimedia Appendix 1). Likewise, 637 Twitter posts were extracted and saved in a CSV file (Multimedia Appendix 2) for further analysis. The obtained
Twitter and Google News texts were supplied to an algorithm written in Python for data cleaning and "normalization" as described by Sahni et al [73] and Salas-Zárate et al [74] with minor modifications. Briefly, all numbers, special characters, punctuations, and symbols except for commas, periods, exclamation marks, question marks, and apostrophes were removed. Repeated white spaces were replaced with a single space followed by the removal of all stop words. In addition, we autocorrected wrongly spelled English words, removed non-English words, and performed lemmatization. The final cleaned corpus was saved into a Pandas dataframe object for further analysis.

\section{Opinion Mining and Sentiment Analysis}

To provide a more symmetrical and global perspective of the emotive patterns in the data sets, two rule- and lexicon-based ML techniques (ie, TextBlob and VADER) and an advance algorithm, which combines a ML model and a neural network (NN) for NLP, (ie, Word2Vec-BiLSTM) were used to process the cleaned data.

\section{TextBlob Sentiment Analysis}

The TextBlob Python library [48] is an easy-to-use publicly available library for NLP tasks such as sentiment analysis, part-of-speech tagging, classification, noun phrase extraction, translation, tokenization, and n-grams. In this study, we used the NaiveBayes analyzer implementation of TextBlob for sentiment analysis, which is a conditional probabilistic classifier implementation as explained by Hasan et al [75] and Singh et al [76]. The sentiment feature of TextBlob makes use of a predefined dictionary classifying positive and negative words and returns two values, polarity and subjectivity. Polarity is a float between -1 and +1 , where -1 means a negative sentiment, +1 means a positive sentiment, and a 0 is considered a neutral sentiment. The subjectivity score is a float between 0 and 1 , and represents the degree of personal opinion or judgement rather than factual information. A subjectivity score closer to 0 implies a more objective view, whereas a score closer to 1 represents a more subjective view. The data is generally supplied as a bag-of-words, and after assigning individual scores to each word, the final sentiment is represented by some pooling of all the sentiments. TextBlob has semantic labels that facilitate fine-grained sentiment analysis by recognizing emojis, emoticons, and punctuations such as exclamation marks.

\section{VADER Sentiment Analysis}

The VADER library [47] is a lexicon- and rule-based sentiment analyzer of the English language constructed from a generalizable, valence-based, human-curated gold standard sentiment lexicon and is available with Python's Natural Language Toolkit library [77]. VADER does not require any training data and is specifically attuned to semantic constructions in social media texts, which are generally informal, yet can be conveniently applied to multiple domains $[77,78]$. A Python algorithm using the VADER library was developed to unveil and categorize the sentiment in our data set. The output of the analysis is a percentage score of positivity, negativity, and neutrality, as well as a compound score. The compound score is a normalized weighted composite score (ie, normalized to be 
between -1 , most extreme negative, and +1 , most extreme positive) that is useful for interpreting the sentiment in a text as a single unidimensional measure of sentiment. On the other hand, the positive, negative, and neutral scores are useful to understand the sentiment in a text in multidimensional measures.

\section{Word2Vec-BiLSTM Sentiment Analysis}

Word2Vec-BiLSTM is an advanced model, which combines ML and AI for NLP. Word2Vec is a ML model that projects natural words, groups of words, or phrases into a multidimensional space, creating vector representations of the word or group of words, which are mapped to vectors of real numbers, otherwise called word embeddings. The resultant two-layer NNs are subsequently trained to reconstruct linguistic contexts of the word or group of words that preserve information about the target word or group of words, the overall context of the word, and the crams variable length sequences to fixed-length vectors. Mikolov et al [79-81] describe in detail the underlying principles and algorithms of Word2Vec. The BiLSTM model $[82,83]$ learns two-way dependencies between time steps of time series data (ie, the output layer gets information from forward and backward states simultaneously), which has presented good results in NLP $[84,85]$. When given adequate training, Word2Vec-BiLSTM models are highly accurate at capturing the ephemeral qualities of human language and guessing the meanings of words within the context of a sentence. The BiLSTM model and related hyperparameters adopted herein with minor modifications is described by Cherniuk [86]. The training data consisted of 75,000 review entries, with a test size of $0.05 \%$ of the training data accordingly labeled as a positive or negative review. The model inputs were feature vectors composed of bigrams and trigrams of the cleaned data set. The model architecture consisted of six layers with a sigmoid activation function, Adam optimizer [87], and binary_crossentropy as the loss function. The model batch size was 100 with an epoch of five. After training, the accuracy of the model was $92 \%$.

\section{Results}

The results of media activity within the study period is summarized in Figure 1 [88-95]. There was relatively low activity on Twitter and Google News about the query terms within the first 3 weeks of the study period (ie, between February 2-23, 2020). Google News features on the search topics soared from 39 news items in the eighth week (ie, March 22, 2020) to 73 news features by the ninth week (ie, March 29, 2020). Similarly to the trend on Google News, Twitter posts increased from 3 to 70 between the eighth and ninth weeks of the study period.

Figure 1. Media activity and sentiment analysis of tweets and Google News headlines and snippets between February 2 and May 5, 2020. (A) Media activity within the study period, (B) TextBlob sentiment analysis, (C) VADER sentiment analysis, and (D) Word2Vec-BiLSTM sentiment analysis. (a) Two French medical doctors made controversial remarks on COVID-19 vaccine testing in Africa on April 1, (b) the World Health Organization strongly remarked about the comments by the French doctors on April 6, (c) Madagascar announced herbal cure to COVID-19 at a virtual meeting of African presidents on April 20, (d) first reported case of COVID-19 in Africa on February 14, and (e) first reported case of COVID-19 in sub-Saharan Africa on February 28. BiLSTM: bidirectional long short-term memory; VADER: Valence Aware Dictionary and Sentiment Reasoner.
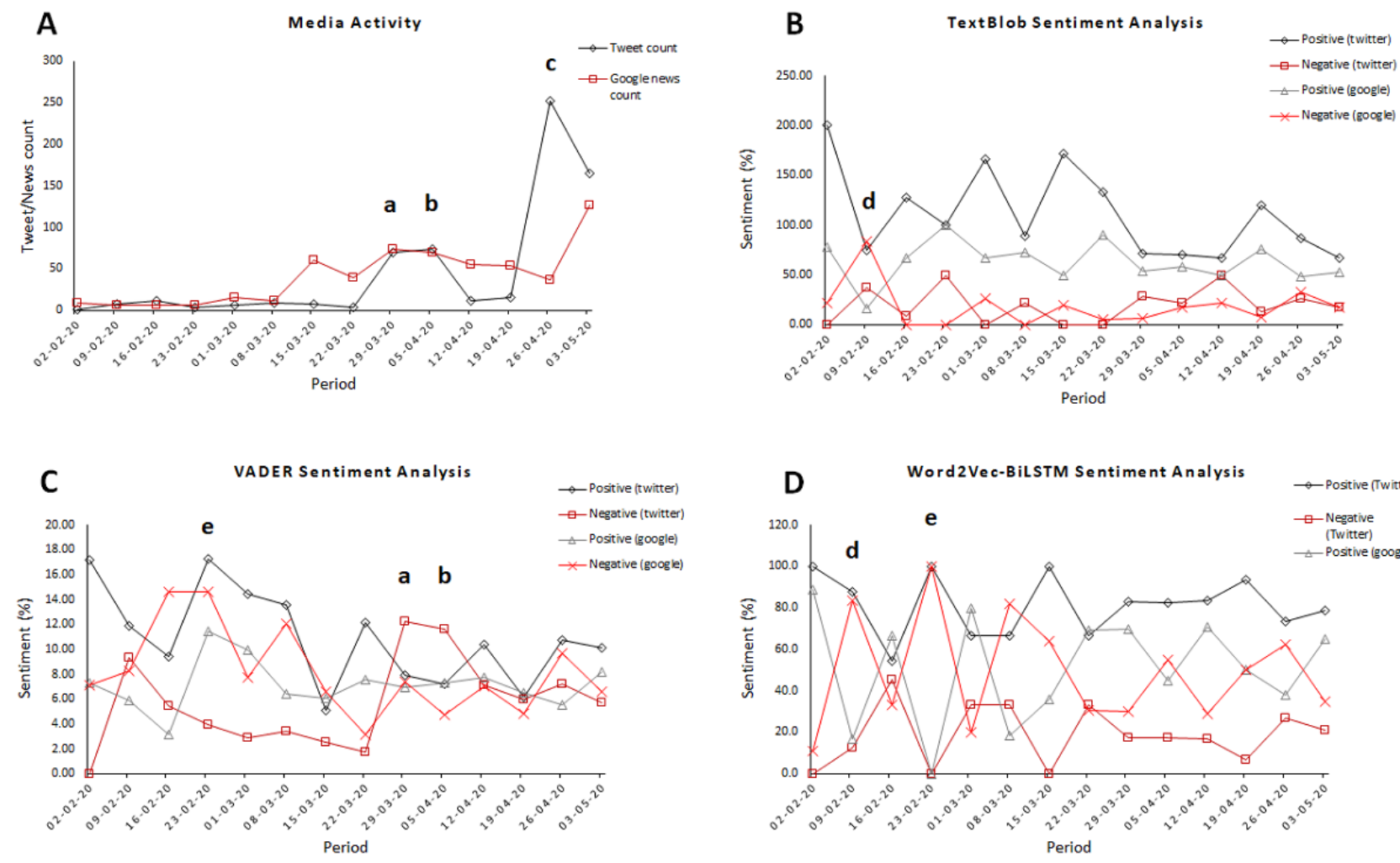

Tables 1 and 2 provide a summary of the sentiment polarities in media communications using computational linguistic models. Zooming in on the results for Google News headlines or

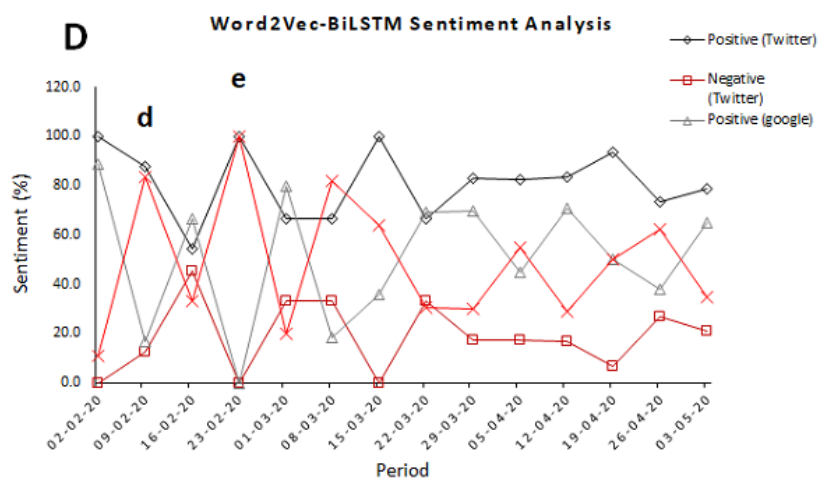

snippets, it can be observed that average polarities from the VADER analysis revealed that the news was $7 \%$ positive, $8 \%$ negative, and $85 \%$ neutral, with a compound score of -2 , which 
indicates overall negativity in the news tonality. The TextBlob and $22 \%$ neutral, with an average subjectivity score of 0.5 , analysis showed that the news was $63 \%$ positive, $19 \%$ negative, indicating a general positivity in the news tonality.

Table 1. Sentiment polarities in media communications (Google News headlines or descriptions) analyzed using the TextBlob, VADER, and Word2Vec-BiLSTM models.

\begin{tabular}{|c|c|c|c|c|c|c|c|c|c|c|}
\hline \multirow[t]{2}{*}{ Period in 2020} & \multicolumn{4}{|c|}{ TextBlob } & \multicolumn{4}{|c|}{ VADER $^{a}$} & \multicolumn{2}{|c|}{ Word2Vec-BiLSTM ${ }^{b}$} \\
\hline & Positive & Negative & Neutral & $\begin{array}{l}\text { Subjectivity } \\
\text { score }\end{array}$ & Positive & Negative & Neutral & $\begin{array}{l}\text { Compound } \\
\text { score }\end{array}$ & Positive & Negative \\
\hline February 2 & 77.78 & 22.22 & 0.00 & 0.48 & 0.07 & 0.07 & 0.86 & 0.02 & 88.89 & 11.11 \\
\hline February 9 & 16.67 & 83.33 & 0.00 & 0.58 & 0.06 & 0.08 & 0.86 & -0.01 & 16.67 & 83.33 \\
\hline February 16 & 66.67 & 0.00 & 33.33 & 0.73 & 0.03 & 0.15 & 0.82 & -0.35 & 66.67 & 33.33 \\
\hline February 23 & 100.00 & 0.00 & 0.00 & 0.58 & 0.11 & 0.15 & 0.74 & -0.11 & 0.00 & 100.00 \\
\hline March 1 & 66.67 & 26.67 & 6.67 & 0.43 & 0.10 & 0.08 & 0.82 & 0.09 & 80.00 & 20.00 \\
\hline March 8 & 72.73 & 0.00 & 27.27 & 0.64 & 0.06 & 0.12 & 0.82 & -0.21 & 18.18 & 81.82 \\
\hline March 15 & 49.18 & 19.67 & 31.15 & 0.40 & 0.06 & 0.07 & 0.87 & -0.04 & 36.07 & 63.93 \\
\hline March 22 & 89.74 & 5.13 & 5.13 & 0.37 & 0.08 & 0.03 & 0.89 & 0.17 & 69.23 & 30.77 \\
\hline March 29 & 53.42 & 6.85 & 39.73 & 0.48 & 0.07 & 0.07 & 0.86 & 0.02 & 69.86 & 30.14 \\
\hline April 5 & 57.97 & 17.39 & 24.64 & 0.43 & 0.07 & 0.05 & 0.88 & 0.07 & 44.93 & 55.07 \\
\hline April 12 & 49.09 & 21.82 & 29.09 & 0.34 & 0.08 & 0.07 & 0.85 & 0.03 & 70.91 & 29.09 \\
\hline April 19 & 75.93 & 7.41 & 16.67 & 0.42 & 0.06 & 0.05 & 0.89 & 0.10 & 50.00 & 50.00 \\
\hline April 26 & 48.65 & 32.43 & 18.92 & 0.46 & 0.06 & 0.10 & 0.85 & -0.14 & 37.84 & 62.16 \\
\hline May 3 & 53.17 & 17.46 & 29.37 & 0.33 & 0.08 & 0.07 & 0.85 & 0.06 & 65.08 & 34.92 \\
\hline Average polarity & 62.69 & 18.60 & 18.71 & 0.48 & 0.07 & 0.08 & 0.85 & -0.02 & 51.02 & 48.98 \\
\hline
\end{tabular}

${ }^{\mathrm{a}}$ VADER: Valence Aware Dictionary and Sentiment Reasoner.

${ }^{b}$ BiLSTM: bidirectional long short-term memory. 
Table 2. Sentiment polarities in media communications (Twitter posts) analyzed using the TextBlob, VADER, and Word2Vec-BiLSTM models.

\begin{tabular}{|c|c|c|c|c|c|c|c|c|c|c|}
\hline \multirow[t]{2}{*}{ Period in 2020} & \multicolumn{4}{|l|}{ TextBlob } & \multicolumn{4}{|l|}{ VADER $^{\mathrm{a}}$} & \multicolumn{2}{|c|}{ Word2Vec-BiLSTM ${ }^{\text {b }}$} \\
\hline & Positive & Negative & Neutral & $\begin{array}{l}\text { Subjectivity } \\
\text { score }\end{array}$ & Positive & Negative & Neutral & $\begin{array}{l}\text { Compound } \\
\text { score }\end{array}$ & Positive & Negative \\
\hline February 2 & 100.00 & 0.00 & 0.00 & 0.92 & 0.17 & 0.00 & 0.83 & 0.70 & 100.00 & 0.00 \\
\hline February 9 & 37.50 & 37.50 & 25.00 & 0.34 & 0.12 & 0.09 & 0.79 & 0.15 & 87.50 & 12.50 \\
\hline February 16 & 63.64 & 9.09 & 27.27 & 0.27 & 0.09 & 0.05 & 0.85 & 0.12 & 54.55 & 45.45 \\
\hline February 23 & 50.00 & 50.00 & 0.00 & 0.37 & 0.17 & 0.04 & 0.79 & 0.48 & 100.00 & 0.00 \\
\hline March 1 & 83.33 & 0.00 & 16.67 & 0.59 & 0.14 & 0.03 & 0.83 & 0.36 & 66.67 & 33.33 \\
\hline March 8 & 44.44 & 22.22 & 33.33 & 0.32 & 0.14 & 0.03 & 0.83 & 0.38 & 66.67 & 33.33 \\
\hline March 15 & 85.71 & 0.00 & 14.29 & 0.52 & 0.05 & 0.02 & 0.92 & 0.16 & 100.00 & 0.00 \\
\hline March 22 & 66.67 & 0.00 & 33.33 & 0.40 & 0.12 & 0.02 & 0.86 & 0.49 & 66.67 & 33.33 \\
\hline March 29 & 38.57 & 27.14 & 34.29 & 0.37 & 0.08 & 0.12 & 0.80 & -0.12 & 82.86 & 17.14 \\
\hline April 5 & 36.49 & 20.27 & 43.24 & 0.35 & 0.07 & 0.12 & 0.81 & -0.11 & 82.43 & 17.57 \\
\hline April 12 & 33.33 & 41.67 & 25.00 & 0.49 & 0.10 & 0.07 & 0.82 & 0.10 & 83.33 & 16.67 \\
\hline April 19 & 66.67 & 6.67 & 26.67 & 0.47 & 0.06 & 0.06 & 0.88 & -0.02 & 93.33 & 6.67 \\
\hline April 26 & 48.02 & 23.41 & 28.57 & 0.37 & 0.11 & 0.07 & 0.82 & 0.11 & 73.41 & 26.59 \\
\hline May 3 & 38.18 & 15.15 & 46.67 & 0.32 & 0.10 & 0.06 & 0.84 & 0.09 & 78.79 & 21.21 \\
\hline Average polarity & 56.61 & 18.08 & 25.31 & 0.43 & 0.11 & 0.06 & 0.83 & 0.21 & 81.16 & 18.84 \\
\hline
\end{tabular}

${ }^{\mathrm{a}}$ VADER: Valence Aware Dictionary and Sentiment Reasoner.

bBiLSTM: bidirectional long short-term memory.

Somewhat similar to the TextBlob results, the Word2Vec-BiLSTM analysis unveiled a slightly more positive sentiment in the news communications, as $51 \%$ of the news was positive and $49 \%$ was negative. On the other hand, taking a look at the results from Twitter, results showed that, for the VADER analysis, the posts were $11 \%$ positive, $6 \%$ negative, and $83 \%$ neutral. The TextBlob analysis showed that the tweets were $57 \%$ positive, $18 \%$ negative, and $25 \%$ neutral, with a subjectivity score of 0.5 , indicating an overall positive sentiment in the tweets. Whereas, the Word2Vec-BiLSTM analysis revealed that $81 \%$ of the tweets were positive, while $19 \%$ were negative, indicating a strong positivity in Twitter communications. Generally, it can be observed that sentiment results from TextBlob were more positive as compared to results from VADER. Elsewhere [96,97], researchers also made similar observations that VADER is more likely to pick up negative tones as compared to TextBlob.

\section{Discussion}

\section{Principal Findings}

Media plays a key role in information dissemination and consumption, which in turn influences people's opinions, attitudes, and decisions. Herein, we leveraged on the capability of computers to comprehend human language and describe emotional polarities in large amounts of data to delineate the sentiments in Twitter and Google News communications within the period of February 2 to May 5, 2020, regarding COVID-19 vaccines in the continent of Africa. In examining the media activity within the stated period, as expected, it was observed that media activity on the subject matter was relatively low in the first 3 weeks of the study period (ie, between February 2-23, 2020; Figure 1). This is because COVID-19 was just beginning to gain media attention in Africa during that time. In fact, the first case of COVID-19 in Africa was reported on February 14, 2020, in Egypt [93], while the first confirmed case in sub-Saharan Africa, Nigeria to be specific, was reported on February 28, 2020 [94].

It can be observed that there was a spike in media activity on both Twitter and Google News between March 29 and April 5, 2020, with corresponding increases in negative sentiments based on results from TextBlob and VADER (Figure 1 and Tables 1 and 2). This period coincides with negative public reception of the remarks made by two French medical doctors on COVID-19 testing in Africa [89]. The director-general of the WHO, Dr Tedros Adhanom Ghebreyesus, in a press briefing on April 6, 2020 , strongly condemned the remarks by the doctors, comments he termed "racist remarks" and ascribed to a "hangover from colonial mentality" [90]. Interestingly coincidental was a public dissent, which culminated in the incineration of a COVID-19 testing facility in Abidjan, capital city of Côte d'Ivoire the same day, on fears that patients with COVID-19 would be treated at the center, because the facility was too close to residential apartments $[98,99]$. This hostile public response is reminiscent of psychosocial effects during the Ebola outbreaks in Central and West Africa wherein some health workers were attacked on suspicion that they were spreading the disease in their communities, rather than providing medical care [98]. Although the event in Abidjan is seemingly an isolated situation, such experiences reiterate the importance of knowledge and 
information consumption during the time of a medical emergency or crisis.

Although there was a generally weak decline in positivity from both Twitter and Google News data across the study period, prevailing sentiments were neutral to positive. This gives an indication that generally truer and more evidence-based information regarding COVID-19 vaccines in Africa are circulating on Twitter and Google News as compared to falsehoods. This is because it is expected that, if factual and more science-based information is circulated on the media platforms about the prospects of a viable vaccine to tackle the COVID-19 pandemic in Africa, the communications and corresponding reactions should possess more positivity than negativity. It was observed that there was generally more positivity in Twitter data as compared to Google News data, which might indicate that there was less falsehoods communicated on Twitter as compared to Google News. This trend seems to be in contrast to observations made by other researchers that fake news spreads faster on social media (eg, Twitter, Facebook, Instagram, Snapchat, and WhatsApp) than evidence-based information [100,101]. Notwithstanding, our findings align considerably to those obtained by Pulido et al [67] on public information about COVID-19, wherein the authors noted that for tweets regarding COVID-19, though false information is tweeted more, it had overall less retweets as compared to fact-checking tweets and that science-based evidence tweets captured more engagement than mere facts. Fung et al [24] also made a similar observation, noting that after the declaration of the Ebola outbreak as an emergency in 2014, more accurate information circulated as compared to false information.

These seemingly counterintuitive findings cannot be logically described by chance or normal human behavior alone. Indeed, our observed sentiment patterns seem to be consistent with deliberate and sustained efforts made on various media platforms like Twitter, Facebook, Google, Microsoft, Reddit, and others to limit the spread of misleading and potentially harmful content regarding the COVID-19 pandemic $[67,102,103]$. For example, in March 2020, Twitter updated its policy guidance to address content sharing on its platform from authoritative sources of global and local health information that goes directly against guidance on COVID-19 [104]. Google, on the other hand, put in place mechanisms to ensure that searches related to COVID-19 on the company's search engine triggered an "SOS Alert," with news from mainstream publications including the WHO, National Public Radio, and the US Centers for Disease Control and Prevention displayed prominently [105].

In consonance with efforts made by the public and social media platforms, other national and international entities and health actors have also intensified efforts to ensure accurate and reliable information to the public regarding COVID-19. For example, the WHO's risk communication team launched a new information platform called WHO Information Network for Epidemics, immediately after it declared COVID-19 a Public
Health Emergency of International Concern, devoted to myth-busting, debunking of false information, and sharing of tailored information with specific target groups [13,67]. In response to skepticism regarding COVID-19 vaccines, Media Monitoring Africa, a media organization that aims to promote the development of critical, ethical, and a free and fair media culture in South Africa and the rest of the continent, triggered its Real411 platform, wherein the public can report disinformation regarding COVID-19 to a digital complaints committee [106]. The platform was originally set up during the country's elections to enable reporting of objectionable speech by members of the public. This strategic media response was deemed necessary following a national survey conducted between April 15-23, 2020, among a representative sample of 600 people regarding COVID-19 vaccines in South Africa, which revealed that $21 \%$ of South Africans were strongly unwilling to be vaccinated [106].

\section{Conclusion}

Information constitutes a critical resource for mitigation and curative measures in the fight against the COVID-19 pandemic. This study assessed prevailing affective inclinations in media submissions regarding COVID-19 vaccines in Africa. Contrary to general perception, the results revealed a more passive to positive sentiment, which we could understand within the context of active media policing in ensuring safe and objective information regarding the COVID-19 pandemic. These findings are consistent with previous studies regarding public information about COVID-19. Our analytical approach attempted to provide a more universal and balanced perspective of the emotive patterns in the data sets by adopting three different NLP models (TextBlob, VADER, and Word2Vec-BiLSTM models) with fundamentally different underlying principles and mechanisms. Of course, sentiment analysis is a complex undertaking because speech communications can be highly subjective. For example, certain words or phrases can have different sentiments (ie, positive or negative) depending on the context of the text. Further to that, some narratives such as ironies and sarcasms are extremely difficult for machines and computers to understand because they cannot be interpreted literally. In this regard, we acknowledge the limitations of our approach (including the scope of the search terms and phrases used to query for the data on the internet); nonetheless, we believe that our study provides a reasonable approximation of the media polarity regarding the study topic. In future undertakings, the Word2Vec-BiLSTM and VADER libraries can be trained using more topic-specific vocabulary to improve their overall accuracies and predictability. Ultimately, building on insights from this study, public health and media actors can be stimulated to develop or re-evaluate relevant policies that promote responsible media use and public consumption to maximize the benefits of health interventions amid the COVID-19 crisis. This does not undermine the efficacy of efforts already made to curb misleading information regarding COVID-19 in the public space. 


\section{Acknowledgments}

This paper was financially supported via the 2020 University Research Committee funding from the University of Johannesburg, granted to the main author.

\section{Conflicts of Interest}

None declared.

\section{Multimedia Appendix 1}

Google News data.

[XLSX File (Microsoft Excel File), 52 KB-Multimedia Appendix 1]

\section{Multimedia Appendix 2}

Twitter data.

[XLSX File (Microsoft Excel File), 99 KB-Multimedia Appendix 2]

\section{References}

1. Driggin E, Madhavan MV, Bikdeli B, Chuich T, Laracy J, Biondi-Zoccai G, et al. Cardiovascular considerations for patients, health care workers, and health systems during the COVID-19 pandemic. J Am Coll Cardiol 2020 May 12;75(18):2352-2371 [FREE Full text] [doi: 10.1016/j.jacc.2020.03.031] [Medline: 32201335]

2. Toure A. COVID-19 (coronavirus) drives sub-Saharan Africa toward first recession in 25 Years. The World Bank. 2020 Apr 09. URL: https://www.worldbank.org/en/news/press-release/2020/04/09/ covid-19-coronavirus-drives-sub-saharan-africa-toward-first-recession-in-25-years [accessed 2020-08-08]

3. Nicola M, Alsafi Z, Sohrabi C, Kerwan A, Al-Jabir A, Iosifidis C, et al. The socio-economic implications of the coronavirus pandemic (COVID-19): a review. Int J Surg 2020 Jun;78:185-193 [FREE Full text] [doi: 10.1016/j.ijsu.2020.04.018] [Medline: 32305533]

4. Martin A, Markhvida M, Hallegatte S, Walsh B. Socio-economic impacts of COVID-19 on household consumption and poverty. Econ Disaster Clim Chang 2020 Jul 23:1-27 [FREE Full text] [doi: 10.1007/s41885-020-00070-3] [Medline: 32838120]

5. Buheji M, da Costa Cunha K, Beka G, Mavrić B, Leandro do Carmo de Souza Y, Souza da Costa Silva S, et al. The extent of COVID-19 pandemic socio-economic impact on global poverty. A global integrative multidisciplinary review. Am J Economics 2020 Aug 1;10(4):213-224. [doi: 10.5923/j.economics.20201004.02]

6. Statement on the second meeting of the International Health Regulations (2005) Emergency Committee regarding the outbreak of novel coronavirus (2019-nCoV). World Health Organization. 2020 Jan 30. URL: https://tinyurl.com/463gxq71 [accessed 2020-05-08]

7. Impact of the coronavirus (COVID-19) on the African economy. Tralac. 2020. URL: https://www.tralac.org/news/article/ 14483-impact-of-the-coronavirus-covid-19-on-the-african-economy.html [accessed 2020-05-08]

8. Sumner A, Hoy C, Ortiz-Juarez E. Estimates of the impact of COVID-19 on global poverty. UNU-WIDER. 2020. URL: https://www.wider.unu.edu/sites/default/files/Publications/Working-paper/PDF/wp2020-43.pdf [accessed 2020-10-12]

9. OECD. OECD Employment Outlook 2020: Worker Security and the COVID-19 Crisis. Paris: OECD Publishing; 2020.

10. Boberg S, Quandt T, Schatto-Eckrodt T, Frischlich L. Pandemic populism: facebook pages of alternative news media and the corona crisis--a computational content analysis. arXiv. Preprint posted online April 6, 2020. [FREE Full text]

11. Cinelli M, Quattrociocchi W, Galeazzi A, Valensise CM, Brugnoli E, Schmidt AL, et al. The COVID-19 social media infodemic. arXiv. Preprint posted online March 10, 2020.

12. Hua J, Shaw R. Corona virus (COVID-19) "Infodemic" and emerging issues through a data lens: the case of China. Int J Environ Res Public Health 2020 Mar 30;17(7) [FREE Full text] [doi: 10.3390/ijerph17072309] [Medline: 32235433]

13. Zarocostas J. How to fight an infodemic. Lancet 2020 Feb 29;395(10225):676 [FREE Full text] [doi: 10.1016/S0140-6736(20)30461-X] [Medline: $\underline{\text { 32113495] }}$

14. Taylor E. New survey reveals lockdown digital usage trends. Stylus. 2020. URL: https://www.stylus.com/ new-survey-reveals-lockdown-digital-usage [accessed 2020-10-12]

15. Kemp S. Report: most important data on digital audiences during coronavirus. TNW. 2020. URL: https://thenextweb.com/ growth-quarters/2020/04/24/report-most-important-data-on-digital-audiences-during-coronavirus/ [accessed 2020-10-12]

16. Rozenblum R, Bates DW. Patient-centred healthcare, social media and the internet: the perfect storm? BMJ Qual Saf 2013 Mar;22(3):183-186. [doi: 10.1136/bmjqs-2012-001744] [Medline: 23378660]

17. Singh S, Dixit A, Joshi G. Is compulsive social media use amid COVID-19 pandemic addictive behavior or coping mechanism? Asian J Psychiatr 2020 Dec;54:102290 [FREE Full text] [doi: 10.1016/j.ajp.2020.102290] [Medline: 32659658] 
18. Sun C, Yang W, Arino J, Khan K. Effect of media-induced social distancing on disease transmission in a two patch setting. Math Biosci 2011 Apr;230(2):87-95 [FREE Full text] [doi: 10.1016/j.mbs.2011.01.005] [Medline: 21296092]

19. Yu W, Liu D, Zheng J, Liu Y, An Z, Rodewald L, et al. Loss of confidence in vaccines following media reports of infant deaths after hepatitis B vaccination in China. Int J Epidemiol 2016 Apr;45(2):441-449. [doi: 10.1093/ije/dyv349] [Medline: 27174834]

20. Jamison AM, Broniatowski DA, Quinn SC. Malicious actors on Twitter: a guide for public health researchers. Am J Public Health 2019 May;109(5):688-692. [doi: 10.2105/AJPH.2019.304969] [Medline: 30896994]

21. Brainard J, Hunter PR. Misinformation making a disease outbreak worse: outcomes compared for influenza, monkeypox, and norovirus. Simulation 2019 Nov 12;96(4):365-374. [doi: 10.1177/0037549719885021]

22. Wang Y, McKee M, Torbica A, Stuckler D. Systematic literature review on the spread of health-related misinformation on social media. Soc Sci Med 2019 Nov;240:112552 [FREE Full text] [doi: 10.1016/j.socscimed.2019.112552] [Medline: $\underline{31561111]}$

23. Betsch C. Advocating for vaccination in a climate of science denial. Nat Microbiol 2017 Jun 27;2:17106. [doi: 10.1038/nmicrobiol.2017.106] [Medline: 28653682]

24. Fung IC, Fu K, Chan C, Chan BSB, Cheung C, Abraham T, et al. Social media's initial reaction to information and misinformation on Ebola, August 2014: facts and rumors. Public Health Rep 2016;131(3):461-473 [FREE Full text] [doi: 10.1177/003335491613100312] [Medline: 27252566]

25. Kim L, Fast SM, Markuzon N. Incorporating media data into a model of infectious disease transmission. PLoS One 2019;14(2):e0197646 [FREE Full text] [doi: 10.1371/journal.pone.0197646] [Medline: 30716139]

26. Wei R, Lo V, Lu H. Third-person effects of health news: exploring the relationships among media exposure, presumed media influence, and behavioral intentions. Am Behav Scientist 2008 Jul 29;52(2):261-277. [doi: 10.1177/0002764208321355]

27. d'Onofrio A, Manfredi P, Manfredi P. Bifurcation thresholds in an SIR model with information-dependent vaccination. Math Modelling Nat Phenomena 2008 Jun 15;2(1):26-43. [doi: 10.1051/mmnp:2008009]

28. Wright J, Polack C. Understanding variation in measles-mumps-rubella immunization coverage--a population-based study. Eur J Public Health 2006 Apr;16(2):137-142. [doi: 10.1093/eurpub/cki194] [Medline: 16207728]

29. Luman ET, Fiore AE, Strine TW, Barker LE. Impact of thimerosal-related changes in hepatitis B vaccine birth-dose recommendations on childhood vaccination coverage. JAMA 2004 May 19;291(19):2351-2358. [doi: 10.1001/jama.291.19.2351] [Medline: 15150207]

30. Lewandowsky S, Ecker UKH, Seifert CM, Schwarz N, Cook J. Misinformation and its correction: continued influence and successful debiasing. Psychol Sci Public Interest 2012 Dec;13(3):106-131. [doi: 10.1177/1529100612451018] [Medline: 26173286]

31. Danovaro-Holliday MC, Wood AL, LeBaron CW. Rotavirus vaccine and the news media, 1987-2001. JAMA 2002 Mar 20;287(11):1455-1462. [doi: 10.1001/jama.287.11.1455] [Medline: 11903035 ]

32. Allcott H, Gentzkow M, Yu C. Trends in the diffusion of misinformation on social media. Res Polit 2019 May 09;6(2):1-8. [doi: 10.1177/2053168019848554]

33. Lazer DMJ, Baum MA, Benkler Y, Berinsky AJ, Greenhill KM, Menczer F, et al. The science of fake news. Science 2018 Mar 09;359(6380):1094-1096. [doi: 10.1126/science.aao2998] [Medline: 29590025]

34. Merino JG. Response to Ebola in the US: misinformation, fear, and new opportunities. BMJ 2014 Nov 07;349:g6712. [doi: 10.1136/bmj.g6712] [Medline: 25380659]

35. Tchuenche JM, Bauch CT. Dynamics of an infectious disease where media coverage influences transmission. Int Scholarly Res Notices 2012 Mar 08;2012:1-10. [doi: 10.5402/2012/581274]

36. Liu R, Wu J, Zhu H. Media/psychological impact on multiple outbreaks of emerging infectious diseases. Computational Math Methods Med 2007;8(3):153-164. [doi: 10.1080/17486700701425870]

37. Yadav S, Ekbal A, Saha S, Bhattacharyya P. Medical sentiment analysis using social media: towards building a patient assisted system. In: Proceedings of the Eleventh International Conference on Language Resources and Evaluation. 2018 Presented at: LREC 2018; May 2018; Miyazaki, Japan.

38. Liu B. Sentiment analysis and opinion mining. In: Hirst G, editor. Synthesis Lectures on Human Language Technologies. San Rafael, CA: Morgan \& Claypool Publishers; 2012.

39. Cambria E, Das D, Bandyopadhyay S, Feraco A. Affective computing and sentiment analysis. In: Cambria E, Das D, Bandyopadhyay S, Feraco A, editors. A Practical Guide to Sentiment Analysis. Cham: Springer; 2017:1-10.

40. Natural language processing for sentiment analysis. Expert System Team. 2016. URL: https://expertsystem.com/ natural-language-processing-sentiment-analysis/ [accessed 2020-05-08]

41. Bollen J, Mao H, Zeng X. Twitter mood predicts the stock market. J Computational Sci 2011 Mar;2(1):1-8. [doi: 10.1016/j.jocs.2010.12.007]

42. Ozturk SS, Ciftci K. A sentiment analysis of twitter content as a predictor of exchange rate movements. Rev Econ Analysis 2014;6(2):140.

43. El Ansari O, Zahir J, Mousannif H. Context-based sentiment analysis: a survey. In: Adbelwahed EH, Bellatreche L, Benslimane D, Golfarelli M, Jean S, Mery D, et al, editors. New Trends in Model and Data Engineering: MEDI 2018 
International Workshops, DETECT, MEDI4SG, IWCFS, REMEDY, Marrakesh, Morocco, October 24-26, 2018, Proceedings. Cham: Springer; 2018:91-97.

44. Kumar A, Garg G. Systematic literature review on context-based sentiment analysis in social multimedia. Multimedia Tools Applications 2019 Feb 23;79(21-22):15349-15380. [doi: 10.1007/s11042-019-7346-5]

45. Nankani H, Dutta H, Shrivastava H, Krishna PVNSR, Mahata D, Shah RR. Multilingual sentiment analysis. In: Agarwal B, Nayak R, Mittal N, Patnaik S, editors. Deep Learning-Based Approaches for Sentiment Analysis. Singapore: Springer; 2020:193-236.

46. Katz G, Ofek N, Shapira B. ConSent: context-based sentiment analysis. Knowledge-Based Syst 2015 Aug;84:162-178. [doi: 10.1016/j.knosys.2015.04.009]

47. Hutto C, Gilbert E. VADER: a parsimonious rule-based model for sentiment analysis of social media text. 2014 Presented at: Eighth International AAAI Conference on Weblogs and Social Media; June 1-4, 2014; Ann Arbor, MI URL: http://comp. social.gatech.edu/papers/icwsm14.vader.hutto.pdf

48. Loria S. TextBlob: Simplified Text Processing. 2020. URL: https://textblob.readthedocs.io/en/dev/ [accessed 2020-05-09]

49. Zhang D, Xu H, Su Z, Xu Y. Chinese comments sentiment classification based on word2vec and SVMperf. Expert Syst Applications 2015 Mar;42(4):1857-1863. [doi: 10.1016/j.eswa.2014.09.011]

50. Bilyk V. Why business applies sentiment anaylsis? 5 successful examples. The App Solutions. 2019. URL: https:/ /theappsolutions.com/blog/development/sentiment-analysis-for-business/ [accessed 2020-10-12]

51. Roccetti M, Marfia G, Salomoni P, Prandi C, Zagari RM, Gningaye Kengni FL, et al. Attitudes of Crohn's disease patients: infodemiology case study and sentiment analysis of Facebook and Twitter posts. JMIR Public Health Surveill 2017 Aug 09;3(3):e51 [FREE Full text] [doi: 10.2196/publichealth.7004] [Medline: 28793981]

52. Greaves F, Ramirez-Cano D, Millett C, Darzi A, Donaldson L. Use of sentiment analysis for capturing patient experience from free-text comments posted online. J Med Internet Res 2013 Nov 01;15(11):e239 [FREE Full text] [doi:

10.2196/jmir.2721] [Medline: 24184993]

53. Abualigah L, Alfar HE, Shehab M, Hussein AMA. Sentiment analysis in healthcare: a brief review. In: Elaziz MA, Al-qaness MAA, Ewees AA, Dahou A, editors. Recent Advances in NLP: The Case of Arabic Language. Cham: Springer; 2020:129-141.

54. García-Díaz JA, Cánovas-García M, Valencia-García R. Ontology-driven aspect-based sentiment analysis classification: an infodemiological case study regarding infectious diseases in Latin America. Future Gener Comput Syst 2020 Nov;112:641-657 [FREE Full text] [doi: 10.1016/j.future.2020.06.019] [Medline: 32572291]

55. Afyouni S, Fetit A, Arvanitis T. \#DigitalHealth: exploring users' perspectives through social media analysis. Stud Health Technol Inform 2015;213:243-246. [Medline: 26153005]

56. Monnier J, Laken M, Carter CL. Patient and caregiver interest in internet-based cancer services. Cancer Pract 2002;10(6):305-310. [doi: 10.1046/j.1523-5394.2002.106005.x] [Medline: 12406053]

57. Melzi S, Abdaoui A, Azé J, Bringay S, Poncelet P, Galtier F. Patient's rationale: patient Knowledge retrieval from health forums. 2014 Presented at: eTELEMED: eHealth, Telemedicine, and Social Medicine; 2014; Barcelone, Spain URL: https:/ hal-lirmm.ccsd.cnrs.fr/lirmm-01130720/document

58. Yang F, Lee AJ, Kuo S. Mining health social media with sentiment analysis. J Med Syst 2016 Nov;40(11):236. [doi: 10.1007/s10916-016-0604-4] [Medline: 27663246]

59. Barkur G, Vibha, Kamath GB. Sentiment analysis of nationwide lockdown due to COVID 19 outbreak: evidence from India. Asian J Psychiatr 2020 Jun;51:102089 [FREE Full text] [doi: 10.1016/j.ajp.2020.102089] [Medline: 32305035]

60. Xue J, Chen J, Chen C, Zheng C, Li S, Zhu T. Public discourse and sentiment during the COVID 19 pandemic: using latent Dirichlet allocation for topic modeling on Twitter. PLoS One 2020;15(9):e0239441 [FREE Full text] [doi: 10.1371/journal.pone.0239441] [Medline: 32976519]

61. Imran AS, Daudpota SM, Kastrati Z, Batra R. Cross-cultural polarity and emotion detection using sentiment analysis and deep learning on COVID-19 related tweets. IEEE Access 2020;8:181074-181090. [doi: 10.1109/access.2020.3027350]

62. Sayce D. The number of tweets per day in 2019. David Sayce. URL: https://www.dsayce.com/social-media/tweets-day/ [accessed 2020-05-08]

63. Genç Ö. The basics of NLP and real time sentiment analysis with open source tools. Towards Data Science. 2019. URL: https://towardsdatascience.com/real-time-sentiment-analysis-on-social-media-with-open-source-tools-f864ca239afe [accessed 2020-05-08]

64. Understanding the infodemic and minsinformation in the fight against COVID-19. IRIS PAHO. 2020. URL: https://iris. paho.org/bitstream/handle/10665.2/52052/Factsheet-infodemic eng.pdf?sequence=14 [accessed 2020-07-09]

65. Haselmayer M, Jenny M. Sentiment analysis of political communication: combining a dictionary approach with crowdcoding. Qual Quant 2017;51(6):2623-2646 [FREE Full text] [doi: 10.1007/s11135-016-0412-4] [Medline: 29070915]

66. Garvey C, Maskal C. Sentiment analysis of the news media on artificial intelligence does not support claims of negative bias against artificial intelligence. OMICS 2020 May;24(5):286-299. [doi: 10.1089/omi.2019.0078] [Medline: 31313979]

67. Pulido CM, Villarejo-Carballido B, Redondo-Sama G, Gómez A. COVID-19 infodemic: more retweets for science-based information on coronavirus than for false information. Int Sociol 2020 Apr 15;35(4):377-392. [doi:

10.1177/0268580920914755] 
68. von Nordheim G, Boczek K, Koppers L. Sourcing the sources: an analysis of the use of Twitter and Facebook as a journalistic source over 10 years in The New York Times, The Guardian, and Süddeutsche Zeitung. Digital Journalism 2018 Sep 20;6(7):807-828. [doi: 10.1080/21670811.2018.1490658]

69. Castillo C, Mendoza M, Poblete B. Information credibility on twitter. In: Proceedings of the 20th International Conference on World Wide Web. 2011 Presented at: WWW '11; March 2011; Hyderabad, India p. 675-684. [doi: 10.1145/1963405.1963500]

70. Mendoza M, Poblete B, Castillo C. Twitter under crisis: can we trust what we RT? In: Proceedings of the First Workshop on Social Media Analytics. 2010 Presented at: SOMA '10; July 2010; Washington DC p. 71-79. [doi: $10.1145 / 1964858.1964869]$

71. Veenstra AS, Iyer N, Hossain MD, Park J. Time, place, technology: Twitter as an information source in the Wisconsin labor protests. Comput Hum Behav 2014 Feb;31:65-72. [doi: 10.1016/j.chb.2013.10.011]

72. Hu H. GoogleNews 1.5.5. The Python Package Index. 2020. URL: https://pypi.org/project/GoogleNews/ [accessed 2020-05-06]

73. Sahni T, Chandak C, Chedeti NR, Singh M. Efficient Twitter sentiment classification using subjective distant supervision. 2017 Presented at: 9th International Conference on Communication Systems and Networks (COMSNETS); January 4-8, 2017; Bangalore URL: https://arxiv.org/pdf/1701.03051.pdf [doi: 10.1109/comsnets.2017.7945451]

74. Salas-Zárate MDP, Medina-Moreira J, Lagos-Ortiz K, Luna-Aveiga H, Rodríguez-García MÁ, Valencia-García R. Sentiment analysis on tweets about diabetes: an aspect-level approach. Comput Math Methods Med 2017;2017:5140631. [doi: 10.1155/2017/5140631] [Medline: 28316638]

75. Hasan A, Moin S, Karim A, Shamshirband S. Machine learning-based sentiment analysis for Twitter accounts. Math Computational Applications 2018 Feb 27;23(1):11. [doi: 10.3390/mca23010011]

76. Kumar Singh A, Kumar Gupta D, Mohan Singh R. Sentiment analysis of Twitter user data on Punjab Legislative Assembly Election, 2017. Int J Modern Education Computer Sci 2017 Sep 08;9(9):60-68. [doi: 10.5815/ijmecs.2017.09.07]

77. Pandey P. Simplifying sentiment analysis using VADER in Python (on social media text). Medium. 2018. URL: https:/ /medium.com/analytics-vidhya/simplifying-social-media-sentiment-analysis-using-vader-in-python-f9e6ec6fc52f [accessed 2020-05-09]

78. Elbagir S, Yang J. Twitter sentiment analysis using natural language toolkit and VADER sentiment. In: Proceedings of the International MultiConference of Engineers and Computer Scientists. 2019 Presented at: IMECS; March 13-15, 2019; Hong Kong URL: https://pdfs.semanticscholar.org/74a2/7879b6c245d9ff7d9c4b41175ffd84b79d73.pdf

79. Mikolov T, Chen K, Corrado G, Dean J. Efficient estimation of word representations in vector space. arXiv. Preprint posted online January 16, 2013. [FREE Full text]

80. Mikolov T, Sutskever I, Chen K, Corrado G, Dean T. Distributed representations of words and phrases and their compositionality. 2013 Presented at: Twenty-seventh Conference on Neural Information Processing Systems; December 5-10, 2013; Lake Tahoe, NV URL: https://papers.nips.cc/paper/

5021-distributed-representations-of-words-and-phrases-and-their-compositionality.pdf

81. Mikolov T, Yih WT, Zweig G. Linguistic regularities in continuous space word representations. 2013 Presented at: 2013 Conference of the North American Chapter of the Association for Computational Linguistics: Human Language Technologies; June 2013; Atlanta, GA URL: https://www.aclweb.org/anthology/N13-1090.pdf

82. Schuster M, Paliwal K. Bidirectional recurrent neural networks. IEEE Trans Signal Processing 1997;45(11):2673-2681. [doi: 10.1109/78.650093]

83. Sharfuddin AA, Tihami MN, Islam MS. A deep recurrent neural network with bilstm model for sentiment classification. 2018 Presented at: 2018 International Conference on Bangla Speech and Language Processing; September 21-22, 2018; Sylhet, Bangladesh. [doi: 10.1109/icbslp.2018.8554396]

84. Rhanoui M, Mikram M, Yousfi S, Barzali S. A CNN-BiLSTM model for document-level sentiment analysis. Machine Learning Knowledge Extraction 2019 Jul 25;1(3):832-847. [doi: 10.3390/make1030048]

85. Ceraj T, Kliman I, Kutnjak M. Redefining cancer treatment: comparison of Word2vec embeddings using deep BiLSTM classification model. University of Zagreb. 2019. URL: https://www.fer.unizg.hr/ download/repository/ TAR-2019-ProjectReports.pdf\#page=16 [accessed 2020-08-09]

86. Cherniuk A. Kaggle. 2019. URL: https://www.kaggle.com/alexcherniuk/imdb-review-word2vec-bilstm-99-acc [accessed 2020-05-09]

87. Kingma DP, Ba J. Adam: a method for stochastic optimization. arXiv. Preprint posted online December 22, 2014.

88. Coronavirus: France racism row over doctors' Africa testing comments. BBC News. 2020. URL: https://www.bbc.com/ news/world-europe-52151722 [accessed 2020-07-13]

89. Bekker L, Mizrahi V. COVID-19 research in Africa. Science 2020 May 29;368(6494):919. [doi: 10.1126/science.abc9528] [Medline: 32467365]

90. COVID-19 virtual press conference - 6 April, 2020. World Health Organization. 2020. URL: https://www.who.int/docs/ default-source/coronaviruse/transcripts/who-audio-emergencies-coronavirus-press-conference-full-06apr2020-final. pdf?sfvrsn=7753b813_2 [accessed 2020-06-16] 
91. Fisayo-Bambi J. Madagascar president with herbal remedy for COVID-19. Africanews. 2020. URL: https://www. africanews.com/2020/04/21/madagascar-president-with-herbal-remedy-for-covid-19-morning-call/ [accessed 2020-06-15]

92. Reihani H, Ghassemi M, Mazer-Amirshahi M, Aljohani B, Pourmand A. Non-evidenced based treatment: an unintended cause of morbidity and mortality related to COVID-19. Am J Emerg Med 2021 Jan;39:221-222 [FREE Full text] [doi: 10.1016/j.ajem.2020.05.001] [Medline: 32402498]

93. Egypt announces first Coronavirus infection. Egypt Today. 2020. URL: https://www.egypttoday.com/Article/1/81641/ Egypt-announces-first-Coronavirus-infection [accessed 2020-06-15]

94. Coronavirus: Nigeria confirms first case in sub-Saharan Africa. BBC News. 2020. URL: https://www.bbc.com/news/ world-africa-51671834 [accessed 2020-06-15]

95. Paintsil E. COVID-19 threatens health systems in sub-Saharan Africa: the eye of the crocodile. J Clin Invest 2020 Jun 01;130(6):2741-2744. [doi: 10.1172/JCI138493] [Medline: $\underline{\text { 32224550] }}$

96. White B. Sentiment analysis: VADER or TextBlob? Toward Data Science. 2020. URL: https://towardsdatascience.com/ sentiment-analysis-vader-or-textblob-ff25514ac540 [accessed 2020-06-16]

97. VADER, IBM Watson or TextBlob: which is better for unsupervised sentiment analysis? Intellica.AI. 2020. URL: https:/ /medium.com/@Intellica.AI/

vader-ibm-watson-or-textblob-which-is-better-for-unsupervised-sentiment-analysis-db4143a39445 [accessed 2020-06-16]

98. Coronavirus: Ivory Coast protesters target testing centre. BBC News. 2020. URL: https://www.bbc.com/news/ world-africa-52189144 [accessed 2020-06-17]

99. Crowd in Ivory Coast destroys coronavirus testing centre in residential area. France 24. 2020. URL: https://www. france24.com/en/20200406-crowd-in-ivory-coast-destroys-coronavirus-testing-centre [accessed 2020-06-17]

100. Vosoughi S, Roy D, Aral S. The spread of true and false news online. Science 2018 Mar 09;359(6380):1146-1151. [doi: 10.1126/science.aap9559] [Medline: 29590045]

101. Fox M. Fake News: Lies spread faster on social media than truth does. NBC News. 2018. URL: https://www.nbcnews.com/ health/health-news/fake-news-lies-spread-faster-social-media-truth-does-n854896 [accessed 2020-07-14]

102. Hancock JR. Las redes sociales y Google intentan contener la desinformación y el pánico sobre el coronavirus. Verne en El País. 2020. URL: https://verne.elpais.com/verne/2020/02/26/articulo/1582728106 118621.html [accessed 2020-06-16]

103. Statt N. Major tech platforms say they're ‘jointly combating fraud and misinformation' about COVID-19. The Verge. 2020. URL: https://www.theverge.com/2020/3/16/21182726/ coronavirus-covid-19-facebook-google-twitter-youtube-joint-effort-misinformation-fraud [accessed 2020-06-16]

104. Roth Y, Pickles N. Updating our approach to misleading information. Twitter Blog. 2020. URL: https://blog.twitter.com/ en_us/topics/product/2020/updating-our-approach-to-misleading-information.html [accessed 2020-06-15]

105. Newton C. Google has been unusually proactive in fighting COVID-19 misinformation. The Verge. 2020. URL: https:/ /www.theverge.com/interface/2020/3/11/21173135/ google-coronavirus-misinformation-youtube-covid-19-twitter-manipulated-media-biden [accessed 2020-06-16]

106. Khumalo J. Poll reveals significant skepticism over possible Covid-19 vaccine. News24. 2020. URL: https://www. news24.com/citypress/Special-Report/Covid-19 Survey/ poll-reveals-significant-skepticism-over-possible-covid-19-vaccine-20200427 [accessed 2020-06-15]

\author{
Abbreviations \\ AI: artificial intelligence \\ API: application processing interface \\ BiLSTM: bidirectional long short-term memory \\ CSV: comma-separated values \\ HepB: hepatitis B \\ ML: machine learning \\ NLP: natural language processing \\ NN: neural network \\ VADER: Valence Aware Dictionary and Sentiment Reasoner \\ WHO: World Health Organization
}


Edited by G Eysenbach; submitted 27.07.20; peer-reviewed by A Chang, M Antoniou; comments to author 28.08.20; revised version received 20.10.20; accepted 08.12.20; published 16.03.21

Please cite as:

Gbashi S, Adebo OA, Doorsamy W, Njobeh PB

Systematic Delineation of Media Polarity on COVID-19 Vaccines in Africa: Computational Linguistic Modeling Study

JMIR Med Inform 2021;9(3):e22916

URL: https://medinform.jmir.org/2021/3/e22916

doi: $\underline{10.2196 / 22916}$

PMID: $\underline{3667172}$

(CSefater Gbashi, Oluwafemi Ayodeji Adebo, Wesley Doorsamy, Patrick Berka Njobeh. Originally published in JMIR Medical Informatics (http://medinform.jmir.org), 16.03.2021. This is an open-access article distributed under the terms of the Creative Commons Attribution License (https://creativecommons.org/licenses/by/4.0/), which permits unrestricted use, distribution, and reproduction in any medium, provided the original work, first published in JMIR Medical Informatics, is properly cited. The complete bibliographic information, a link to the original publication on http://medinform.jmir.org/, as well as this copyright and license information must be included. 